Published in: High Press. Res. 40, 88 (2020)

\title{
A nano-polycrystalline diamond anvil cell with bulk metallic glass cylinder for single-crystal neutron diffraction
}

Keishiro Yamashita ${ }^{\text {a*, Kazuki Komatsu }}{ }^{\mathrm{a}}$, Stefan Klotz ${ }^{\mathrm{b}}$, Maria T.

Fernández-Díaz ${ }^{c}$, Oscar Fabelo ${ }^{\mathrm{c}}$, Tetsuo Irifune ${ }^{\mathrm{d}}$, Kazumasa Sugiyama ${ }^{\mathrm{e}}$, Tooru Kawamata ${ }^{\mathrm{e}}$, Hiroyuki Kagi ${ }^{\mathrm{a}}$

${ }^{a}$ Geochemical Research Center, Graduate School of Science, The University of Tokyo, Tokyo, Japan; ${ }^{b} I M P M C$, UMR 7590, Sorbonne Université, Paris, France; ${ }^{c}$ Institut Laue-Langevin, Grenoble, France; ${ }^{d}$ Geodynamics Research Center, Ehime University, Matsuyama, Japan; ${ }^{e}$ Institute for Materials Research, Tohoku University, Sendai, Japan

Corresponding author. Email: k1c.yamas@eqchem.s.u-tokyo.ac.jp 


\title{
A nano-polycrystalline diamond anvil cell with bulk metallic glass cylinder for single-crystal neutron diffraction
}

\begin{abstract}
A high-pressure cell for in-situ single-crystal neutron diffraction was developed. The cell uses nano-polycrystalline diamond anvils in a tubular load frame made of bulk metallic glass which is highly transparent to neutrons and does not produce Bragg reflections. Diffraction peaks from a sample can be measured from almost any direction and the simple geometry of the cell allows accurate attenuation corrections. We demonstrate the operation of the cell by ambientpressure experiment using a single-crystal of $\mathrm{NaCl}$ on the $\mathrm{D} 9$ diffractometer at the Institute-LaueLangevin. A high-pressure experiment was also carried out on a single crystal of ice VII at 2.35 GPa showing the potential to detect weak diffraction spots. The correct integration of weak reflections together with the simple attenuation correction will help to carry out precise structure analysis and address new scientific problems using neutron diffraction.
\end{abstract}

Keywords: nano-polycrystalline diamond, bulk metallic glass, single-crystal neutron diffraction, high-pressure apparatus

\section{Introduction}

Neutron diffraction is a fundamental and powerful technique to obtain structural information on crystals. It allows us to observe magnetic properties and low- $Z$ atoms, particularly hydrogen positions in materials, whereas they are not straightforwardly observable by x-ray diffraction.

Even though sophisticated analytical techniques for powder diffraction have been developed, it has a significant disadvantage for structure refinements: the reduction of information from three dimensions to one dimension results in severe peak overlap which 
becomes more serious under high pressure owing to peak broadening. In contrast, singlecrystal diffraction measurements provide us more direct structural information. Furthermore, several orders of magnitude stronger intensities from a single crystal, compared with those from powder diffraction in a limited detector region around Bragg spots facilitates considerably the detection of weak signals.

In spite of its importance, single-crystal neutron diffraction measurements under high-pressure conditions are still technically challenging by reason of a large highpressure cell surrounding a small sample. Although it is necessary to measure as many as possible Bragg reflections in order to increase the completeness, it is usually difficult to measure diffracted neutrons passing through the high-pressure cell on account of the large attenuation of the various components (anvils, cell body, and so on). The pair of singlecrystal diamonds that are usually used as anvils for opposed type high-pressure cells also hinder the indexing of sample peaks because of the extremely strong Bragg reflections from the diamonds.

Bull et al. [1] used the Paris-Edinburgh press, a widely-used press for its easiness of handling [2], for single-crystal neutron diffraction of $\mathrm{KD}_{2} \mathrm{PO}_{4}$ and squaric acid $\left(\mathrm{D}_{2} \mathrm{C}_{4} \mathrm{O}_{4}\right)$ at 5, 7.5 GPa. Further high-pressure data were collected on squaric acid at 3.5 and $10 \mathrm{GPa}$ [3]. The sample space in the PE-press is surrounded by two-opposed anvils and two or four supporting pillars which limit the accessible reciprocal space only in a horizontal plane perpendicular to the compression axis.

Binns et al. [4] succeeded in measuring almost all diffraction peaks from single crystals of hexamethylenetetramine $\left(\mathrm{C}_{6} \mathrm{H}_{12} \mathrm{~N}_{4}\right)$ and L-Arginine $\left(\mathrm{C}_{6} \mathrm{H}_{14} \mathrm{~N}_{4} \mathrm{O}_{2} \cdot 2 \mathrm{H} 2 \mathrm{O}\right)$ in a miniature Merrill-Bassett type diamond-anvil cell. This cell was made of a berylliumcopper alloy body and Boehler-Almax type diamond anvils. The applied pressure was 
less than $0.3 \mathrm{GPa}$ but the cell is expected to achieve $5 \mathrm{GPa}$. The data resulted in high completeness values after masking parasitic scattering from the single-crystal diamonds. Haberl et al. [5] used SNAP DACs [6,7] with polycrystalline diamond (Versimax ()) for single crystal diffraction of $\mathrm{MnP}$ up to $10 \mathrm{GPa}$. Higher pressure was accomplished using multi-carat single-crystal diamond anvils grown by Chemical Vapor Deposition (CVD) for $\mathrm{KD}_{2} \mathrm{PO}_{4}$. The cell was similar to the formerly developed wide-angle and SNAP DACs [6,7] and consists of CuBe parts, except for the anvils and the steel gasket, for its non-magnetic properties and superior thermal conductivity in view of the combined high magnetic field and ultra-low temperature. Its wide opening angle of $\pm 60^{\circ}$ is achieved by large cutouts on the side of conical anvils which were improved to be more stable than the previous ones.

Grzechnik et al. (2018) [8] carried out high-quality diffraction measurements on $\mathrm{MnFe}_{4} \mathrm{Si}_{3}$ by using a modified panoramic DAC. Their cell also contained large cutouts with wide opening angles (horizontal: $135^{\circ}$, vertical: $80^{\circ}$ ) next to the side of BoehlerAlmax type diamond anvils. A CuBe gasket with a thickness of more than $1 \mathrm{~mm}$ and a diameter of $8 \mathrm{~mm}$ as well as the data collection with a point detector seemed to contribute to suppressing the parasitic scatterings from the single-crystal diamonds. Its attenuation correction was quite simple since the neutron beams only passed through the gasket.

The problem in all the various cell-designs is related to the question of whether it is preferred to allow neutrons passing through bulky cell materials and then correct diffraction intensities, or to avoid correction procedures by adopting a design with as wide as possible opening angles. Most cell construction materials (steels and other alloys) have low neutron transmittance. Recently, several interesting materials were reported and regarded as promising candidates for neutron diffraction studies: nano-polycrystalline diamond (NPD) and bulk metallic glass (BMG). 
NPD is a sintered material of nano-polycrystalline diamonds which is harder and tougher than a single-crystalline diamond [9]. NPD does not produce Bragg spots which makes the identification of single-crystal sample reflections much easier. Attenuation correction is also much simpler than in the case of single-crystal diamonds, whose attenuation is complicated depending on the incident and scattering angle with respect to the crystal orientation of diamond anvils.

BMG consists of several metallic elements, has a completely amorphous structure, and has higher tensile strength than normal metals [10]. The attenuation coefficient of $\mathrm{Zr}-$ based BMG is less than $0.05 \mathrm{~mm}^{-1}$ owing to the very low total cross-section of $\mathrm{Zr}$. Komatsu et al. used Zr-based BMG as a cylinder material for piston-cylinder highpressure cells instead of $\mathrm{CuBe}$ alloy or other metals [11]. Its potential applicability for measurements of magnetic properties under pressure is also supposed, for its magnetic susceptibility is comparable to most of $\mathrm{CuBe}$ alloys.

Here we report a new high-pressure cell for measuring neutron diffraction from a single crystal sample without geometrical limitations. The cell has optical access along the thrust axis since NPD is transparent, even as mm-size anvils. This is an important issue since visual access to the sample allows monitoring the state of the single crystal and avoid damaging it during the compression procedure. Also, many systems of interest, in particular, hydrogen-bonded solids like ice VI and VII, can only be grown under pressure, i.e. in situ, by carefully adjusting pressure and temperature. Optical access to the sample in such cases is mandatory.

\section{Experiments}

\section{Cell design}

The essential feature of the high-pressure cell is that it spatially separates the neutron 
transparent parts from the mechanical loading mechanism (Fig. 1). The sample is located on a metallic tip consisting only of the NPD anvils, a gasket, and a BMG cylinder. The load onto the anvils is applied by an M52 screw and transferred to the lower anvil by a thin piston tube made of WC. On the very end of the BGM tube is an M6 nut made of WC which serves as a backing seat for the NPD anvil. The NPD anvils have $\phi 1.5$ culet with a $45^{\circ}$ taper. Circular TiZr and $\mathrm{CuBe}$ gaskets of diameter $3.8 \mathrm{~mm}$ were used for ambient-pressure and high-pressure experiments respectively. $\phi 0.8 \mathrm{~mm}$ hole was drilled on the plates as a sample space after preindentation.

\section{Sample observation}

The sample space can be optically observed through the NPD anvil with a microscope from the top of the cell. Two types of NPD anvil pairs were prepared, one with a flat culet and the other with a $\phi=1 \mathrm{~mm}$ cup. The cupped anvils are known to be beneficial to increase the sample volume and to prevent gasket flow at higher pressure [12], but it is difficult to see the sample. The cupped anvils were used for the ambient-pressure tests where there is no need for optical access. The WC piston tube has a hole with a diameter of $1 \mathrm{~mm}$ in order to introduce an optical fibre as a light source. The flat anvils were used for high-pressure experiments. The sample pressure can be measured by the ruby fluorescence method [13]. This is necessary to control the pressure in order to grow single crystals from a solution. 


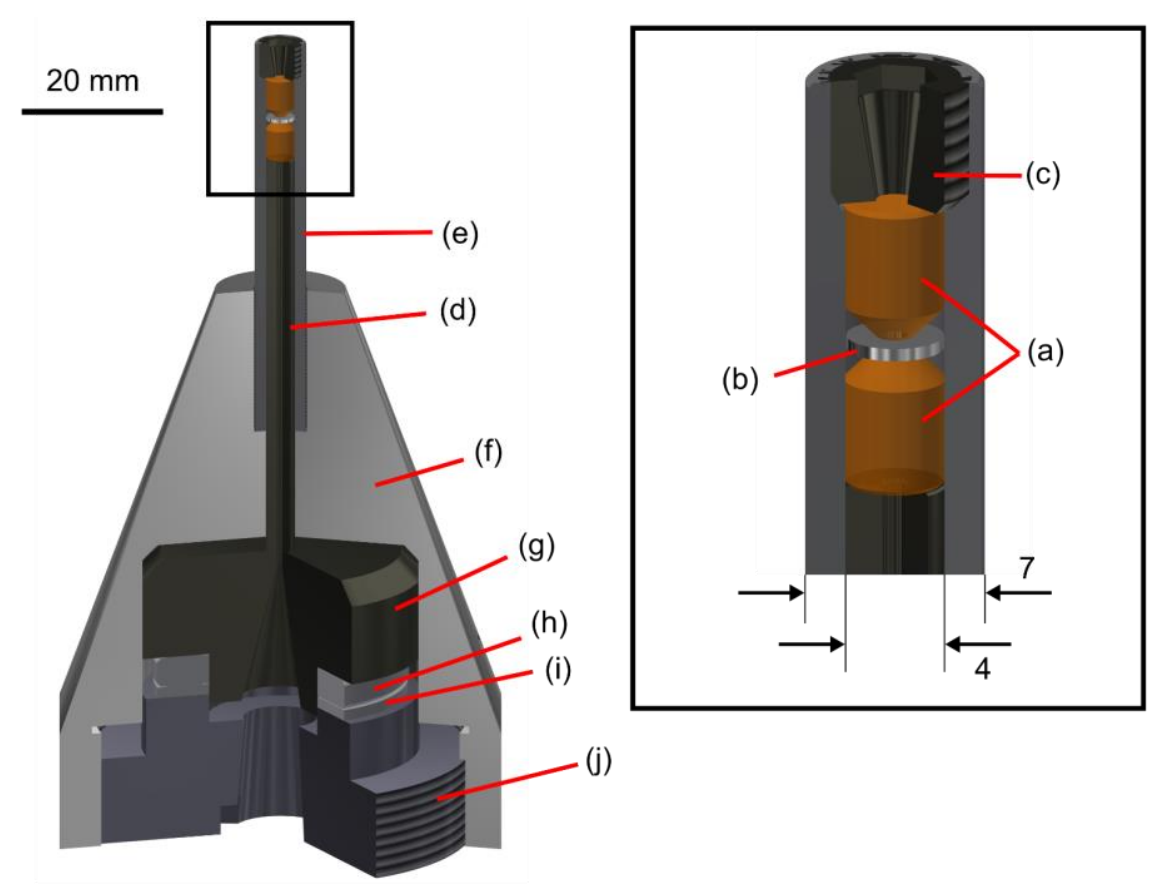

Figure 1. Schematic image of the high-pressure cell; (a) anvils (NPD), (b) gasket, (c) back nut (WC), (d) piston tube (WC), (e) cylinder (Zr-based BMG), (f) body (SUS420), (g) piston (WC), (h) bearing, (i) spacer, (j) back screw (SUS304). The inset shows an expanded image of the tip of the cell.

\section{NaCl at ambient pressure}

Sodium chloride $(\mathrm{NaCl})$ was used as the first sample of our experiment. It has a cubic symmetry $(F m \overline{3} m)$ and produces a small number of strong diffraction spots. $\mathrm{NaCl}$ crystals were recrystallised by slow cooling in a saturated aqueous solution from $60{ }^{\circ} \mathrm{C}$ to room temperature. Ambient-pressure experiments with and without the high-pressure cell were conducted to evaluate the attenuation and background produced by the cell. $\mathrm{NaCl}$ crystals of comparable dimensions $0.57 \times 0.76 \times 0.6 \mathrm{~mm}($ crystal A) and $0.62 \times 0.54 \times 0.35 \mathrm{~mm}$ (crystal B) were selected from the recrystallised crystals. Crystal A was mounted on a vanadium pin with a diameter of $1 \mathrm{~mm}$ while crystal $\mathbf{B}$ was inserted on the NPD anvil with the $\phi=1 \mathrm{~mm}$ cup using a small amount of adhesive. A TiZr gasket was placed between the two anvils and the screws were tightened to reproduce the cell geometry in high-pressure experiments. This TiZr gasket with an initial thickness of $0.65 \mathrm{~mm}$ was 
preindented to $0.41 \mathrm{~mm}$.

\section{Single crystal growth of deuterated ice VII}

A high-pressure experiment was carried out to grow a single crystal of deuterated ice VII from a MeOD:EtOD: $\mathrm{D}_{2} \mathrm{O}=4: 1: 2$ mixture. Ice VII is a polymorph that is stable above 2 $\mathrm{GPa}$ at room temperature. The mixture was loaded into the sample space with a $\mathrm{CuBe}$ gasket of thickness $0.45 \mathrm{~mm}$ preindented from an initial thickness of $0.77 \mathrm{~mm}$. The solution crystallised at $2.43 \mathrm{GPa}$ and a single crystal of ice VII was obtained at $2.35 \mathrm{GPa}$.

\section{Neutron diffraction on the $D 9$ single-crystal diffractometer at the ILL}

Single-crystal neutron diffraction experiments were conducted on D9 which is a fourcircle diffractometer at ILL equipped with a two-dimensional multidetector using monochromatic neutrons. The incident neutron beam $(\lambda=0.8365 \AA)$ was collimated by a $\mathrm{cBN}$ disk hole with a diameter of $2 \mathrm{~mm}$. The data collection consisted of omega or omegagamma scans with fixed $\phi$ and $\chi$ angles, for those reflections at high-Q. The relation of rotation and high-pressure cell geometry is described in Figure 2. 31 points were collected in each scan with a width adapted to the instrument resolution function $\left(\right.$ e.g. $\Delta \omega=1.6^{\circ}$ for $\theta=15-21^{\circ}$ ) and the crystal mosaicity, with a counting time of 5 seconds/point for the sample glued on the vanadium pin and $10 \mathrm{~s} /$ point for the sample placed in the pressure cell. Since it takes much time to obtain data at D9's maximum resolution, not all reflections were collected in this experiment. Both data accumulation times spent a similar time, i.e. ca. 19 and 20 hours for $\mathbf{A}$ and $\mathbf{B}$, respectively. The listed data were corrected as described in the following chapter. The diffraction patterns from $\mathrm{NaCl}$ with enough intensities were analysed using SHELXL [14] (Table 1). 


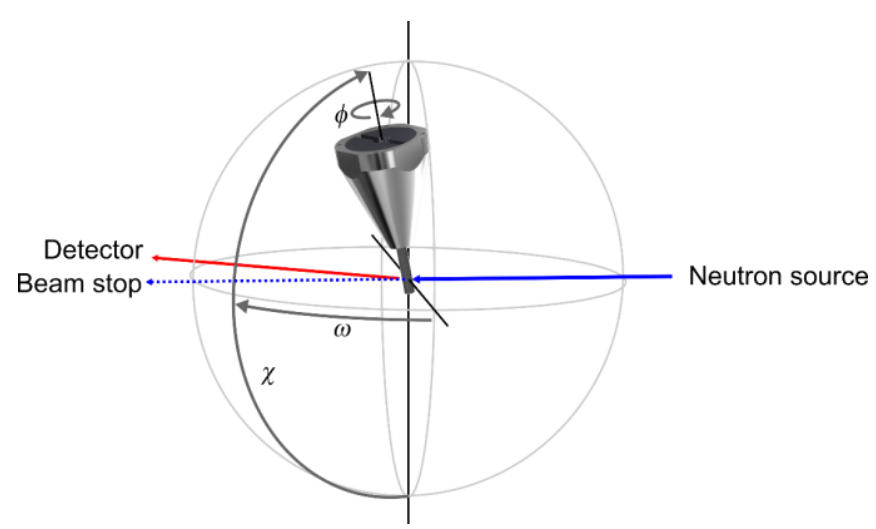

Figure 2. Schematic image of diffraction geometry. The high-pressure cell was mounted on the $\phi$ circle of the Eulerian Cradle on the D9. The blue and red lines indicate beam paths of an incident and a diffracted neutron respectively.

\section{Geometrical-based attenuation correction}

The attenuation by the high-pressure cell can be calculated by a function determined by only one parameter describing the inclination of the cylinder axis since the cell is almost completely rotational symmetric around the sample. The transmittances were higher than $80 \%$ unless the incident or diffracted beam passes through the back nut made of WC which attenuates neutrons at least twice as much as BMG and NPD. Nevertheless, the lowest transmittance was always over $50 \%$.

\section{Results and discussion}

\section{Test study with $\mathrm{NaCl}$}

The statistics of the data for $\mathrm{NaCl}$ at ambient pressure in and out of the high-pressure cell is summarised in Table 1 . The crystal sizes of $\mathrm{NaCl}$ were almost on the lower limit for usable samples on D9. It is notable that low background noise and the absence of any Bragg spots except the sample made it easy to identify sample peaks as well as index the reflections using the dedicated $32 \times 32$ pixels $(64 \times 64 \mathrm{~mm})$ D9-detector. Only Debye rings from the NPD anvils were observed but these can be easily subtracted using conventional 
peak-fitting procedures. The $R_{\text {int }}$ value of raw data of $\mathbf{B}$ was 0.1235 , much larger than that of $\mathbf{A}$, and this was mainly caused by the attenuation by the WC back nut on the top of the BMG cylinder. The attenuation-corrected $R_{\text {int }}$ value decreased to 0.1100 , comparable with the $R_{\sigma}$. The $R_{\sigma}$ comes from the intensity variation of each reflection and can be the lower limit of the $R_{\text {int. }}$ The $R_{\sigma}$ can be improved by increasing the exposure time in the actual measurements for precise structural analysis. These statistical analyses do not contain complicated corrections such as masking parasitic scatterings arising from single-crystal diamonds but only the simple geometrical-based attenuation correction. Complicated correction procedures have risks to lead to unexpected errors and miss out signs of slight changes that can be easily masked by many intense peaks from diamonds.

Table 1. Crystallographic data for $\mathrm{NaCl}$.

\begin{tabular}{lcc} 
& $\mathbf{A}_{\text {out of the cell }}$ & $\mathbf{B}_{\text {in the cell }}$ \\
\hline Crystal size $\left(\mathrm{mm}^{3}\right)$ & $0.57 \times 0.76 \times 0.6$ & $0.62 \times 0.54 \times 0.35$ \\
$a(\AA)$ & $5.639(9)$ & $5.642(3)$ \\
Total reflections & 204 & 180 \\
Used reflections $(I>2 \sigma)$ & 158 & 150 \\
Unique reflections $(I>2 \sigma)$ & 38 & 54 \\
Range of $h, k, l$ & $-8 \leq h \leq 8$ & $-10 \leq h \leq 2$ \\
& $0 \leq k \leq 8$ & $0 \leq k \leq 10$ \\
$R_{\text {int }}$ & $-3 \leq l \leq 9$ & $0 \leq l \leq 10$ \\
$R_{\sigma}$ & 0.0936 & 0.1100 \\
$R_{1}$ & 0.0718 & 0.1167 \\
$w R_{2}$ & 0.0515 & 0.0689 \\
$S$ & 0.1231 & 0.1336 \\
& 0.896 & 0.842
\end{tabular}

\section{Ice VII}

A single crystal of ice VII was grown in the high-pressure cell at room temperature with gradually increasing pressure up to $2.35 \mathrm{GPa}$ (Fig. 3(a)). One diffraction peak was observed with an exposure time of 2 seconds (Fig. 3(b)), which should correspond to the 110 reflection with a $d$-spacing of $2.33 \AA$. Unfortunately, the BMG cylinder broke during scanning after several hours at the edge of the thread for the WC back nut (this deficiency 
of the cell was corrected meanwhile). We note again that no Bragg spots from the highpressure cell were observed which means that the observed peak undoubtedly originated from ice VII.
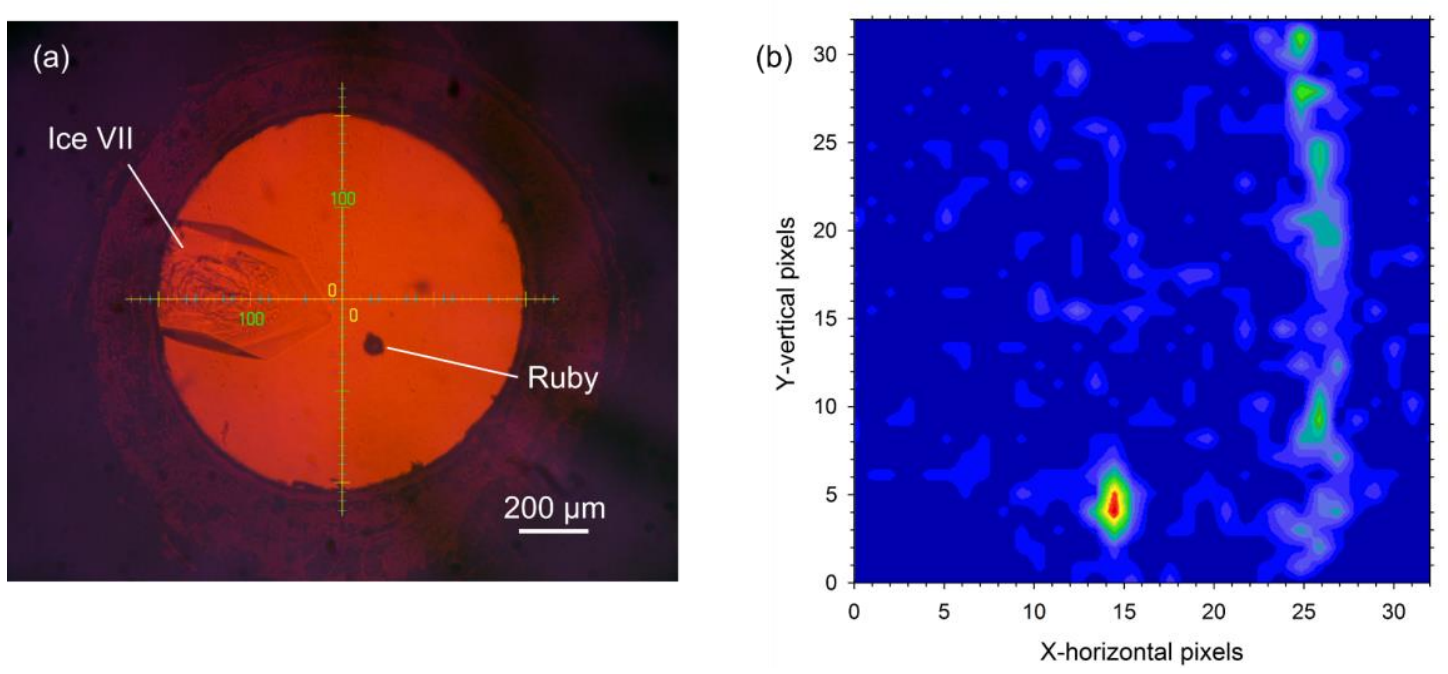

Figure 3. (a) Photograph of ice VII grown in the high-pressure cell at room temperature and 2.35 GPa. (b) A peak from the singe crystal of ice VII in a two-dimensional neutron diffraction pattern with an exposure time of 2 seconds. The image (a) is reddish because of a notch filter in the optics and the yellowish colour of NPD.

\section{Concluding remarks}

We developed a high-pressure cell for in-situ single-crystal neutron diffraction using NPD anvils and BMG as part of the load frame. Its design enables to observe as many diffraction spots as possible without serious background problems such as parasitic scattering from single-crystal diamonds. The cell has also very low neutron attenuation. Test experiments on single-crystalline $\mathrm{NaCl}$ on the D9 diffractometer at ILL demonstrated low background levels and absence of Bragg peaks not from the sample. The cell will hopefully promote single-crystal neutron diffraction and is particularly useful for the analysis of complex crystal structures. While the cell still needs to be 
improved for studies at higher pressures, the concept of this cell is expected to be advantageous for structure refinements from the in-situ high-pressure experiment.

\section{Acknowledgements}

Neutron diffraction experiments were performed through the ILL user programs (No. 515-624). This study was supported by JSPS KAKENHI (Grant Numbers: 18H05224, 18H01936, 15H05829) and the JSPS-CNRS bilateral joint research grant PRC2191.

\section{References}

[1] Bull CL, Guthrie M, Nelmes RJ, et al. Time-of-flight single-crystal neutron diffraction to 10GPa and above. High Press. Res. 2009;29:780-791.

[2] Klotz S, Hamel G, Frelat J. A new type of compact large-capacity press for neutron and X-ray scattering. High Press. Res. 2004;24:219-223.

[3] Bull CL, Guthrie M, Archer J, et al. High-pressure single-crystal neutron diffraction to $10 \mathrm{GPa}$ by angle-dispersive techniques. J. Appl. Crystallogr. 2011;44:831-838.

[4] Binns J, Kamenev K V., McIntyre GJ, et al. Use of a miniature diamond-anvil cell in high-pressure single-crystal neutron Laue diffraction. IUCrJ. 2016;3:168179.

[5] Haberl B, Dissanayake S, Wu Y, et al. Next-generation diamond cell and applications to single-crystal neutron diffraction. Rev. Sci. Instrum. 2018;89:092902.

[6] Boehler R, Molaison JJ, Haberl B. Novel diamond cells for neutron diffraction using multi-carat CVD anvils. Rev. Sci. Instrum. 2017;88;083905.

[7] Haberl B, Dissanayake S, Ye F, et al. Wide-angle diamond cell for neutron scattering. High Press. Res. 2017;37:495-506.

[8] Grzechnik A, Meven M, Friese K. Single-crystal neutron diffraction in diamond 
anvil cells with hot neutrons. J. Appl. Crystallogr. 2018;51:351-356.

[9] Irifune T, Kurio A, Sakamoto S, et al. Ultrahard polycrystalline diamond from graphite. Nature 2003;421:599-600.

[10] Yokoyama Y, Tokunaga H, Yavari AR, et al. Tough Hypoeutectic Zr-Based Bulk Metallic Glasses. Metall. Mater. Trans. A. 2011;42:1468-1475.

[11] Komatsu K, Munakata K, Matsubayashi K, et al. Zr-based bulk metallic glass as a cylinder material for high pressure apparatuses. High Press. Res. 2015;35:254262.

[12] OSAKABE T, YAMAUCHI H, OKUCHI T. Development of High-Pressure Technique for Single-Crystal Magnetic Neutron Diffraction under $10 \mathrm{GPa}$. Rev. High Press. Sci. Technol. 2010;20:72-75.

[13] Mao HK, Bell PM, Shaner JW, et al. Specific volume measurements of $\mathrm{Cu}$, Mo, $\mathrm{Pd}$, and $\mathrm{Ag}$ and calibration of the ruby $\mathrm{R} 1$ fluorescence pressure gauge from 0.06 to 1 Mbar. J. Appl. Phys. 1978;49:3276-3283.

[14] Sheldrick GM. Crystal structure refinement with SHELXL. Acta Crystallogr. Sect. C Struct. Chem. 2015;71:3-8. 